\title{
Application de l'ultrafiltration \\ à la fabrication de lait concentré sucré
}

\author{
par \\ D. SERPA ALVAREZ*, M. BENNASAR \\ et B. TARODO DE LA FUENTE \\ Laboratoire de Technologie alimentaire \\ Université des Sciences et Techniques du Languedoc \\ place E.- Bataillon - 34060 Montpellier cedex
}

\section{INTRODUCTION}

Dès le début de la fabrication du lait concentré sucré les techniciens se sont trouvés confrontés avec le problème de la cristallisation inévitable [1] du lactose lors du refroidissement du produit à la sortie des appareils d'évaporation. En effet, alors que la solubilité du lactose dans une solution de saccharose à 62 Brix n'est que de $16,9 \mathrm{~g}$ pour $100 \mathrm{~g}$ d'eau à $30^{\circ} \mathrm{C}$ [2], sa teneur dans un lait concentré sucré atteint 50 p. 100 dans les mêmes conditions. Dans ce cas, si la cristallisation du lactose n'est pas contrôlée, le produit fini a toutes les chances de présenter une texture sableuse qui n'est pas acceptée par les consommateurs.

Nickerson et al. $[3,4,5,6,7,8,9,10,11]$, Jelen et Coulter $[12,13]$ et Thurbly et Sitnai [14] ont étudié les différents paramètres qui conditionnent cette cristallisation. Les premières fabrications ont été réalisées en contrôlant la vitesse de refroidissement, qui doit être très rapide jusqu'à $30^{\circ} \mathrm{C}$ et plus lente ensuite, puis en ajoutant du lactose finement pulvérisé (150 à $200 \mathrm{~g}$ par tonne) [1], pour obtenir une vitesse de nucléation plus grande que la vitesse de croissance des cristaux qui doivent rester de petite taille [15]. Actuellement, le refroidissement et l'addition de lactose sont utilisés conjointement mais on trouve encore parfois une texture sableuse dans certaines fabrications commerciales.

* Adresse actuelle : Instituto Universitario de Tecnologia de Cumana. Departamento de Biologia Aplicada. Apartado 255. Cumana-Estado Sucre (Venezuela). 
D'autres chercheurs ont essayé de jouer sur la concentration en lactose du produit fini. C'est ainsi que des procédés venant s'ajouter au schéma classique de fabrication et permettant de diminuer la quantité de sucre par hydrolyse enzymatique $[16,17,18]$ ou cristallisation et élimination des cristaux [2] ont donné des résultats intéressants.

Dans cet article nous exposerons les essais que nous avons entrepris pour déterminer les conditions optimales de fabrication du lait écrémé concentré sucré par ultrafiltration, procédé qui présente l'avantage de concentrer le produit et d'éliminer en même temps suffisamment de lactose pour éviter la cristallisation lors du refroidissement et du stockage.

\section{MATERIEL ET METHODES}

\section{Ultrafiltration}

Elle est réalisée avec un module Amicon 202 équipé de membranes Diaflo PM 30. La pression de travail de 3,8 bars est assurée par de l'azote.

Dans la première partie de notre étude le lait écrémé a été pasteurisé 15 secondes à $90^{\circ} \mathrm{C}$ avec un pasteurisateur A.P.V. Junior, et concentré à 50 et $60^{\circ} \mathrm{C}$ pour étudier l'influence de ces deux températures sur l'ultrafiltration des matières azotées, du calcium et du Iactose. Des fractions d'ultrafitrat ont été recueillies et analysées pour des valeurs différentes du facteur de concentration $F$ défini par le rapport : volume de lait initial / volume du rétentat.

Dans la deuxième partie nous avons concentré le même lait à différentes valeurs du facteur $\mathrm{F}$ et nous lui avons ajouté du saccharose à raison de $170 \mathrm{~g}$ par litre de liquide initial; ces fabrications ont été analysées et comparées à un lait écrémé concentré sucré du commerce.

Dans la troisième partie nous avons ultrafiltré à $50^{\circ} \mathrm{C}$ jusqu'à un facteur $\mathrm{F}$ égal à 5 des laits pasteurisés 30 secondes à 90,100 et $115^{\circ} \mathrm{C}$, et nous les avons additionnés de saccharose comme précédemment. Nous avons mesuré la viscosité des produits concentrés pour étudier l'influence des barèmes de pasteurisation sur ce paramètre, ainsi que les quantités de calcium et de lactose éliminées. Dans le cas du lait concentré préparé à partir du lait pasteurisé à $115^{\circ} \mathrm{C}$ nous avons également mesuré le rapport calcium / phosphore et recherché la présence de cristaux de lactose.

\section{Lactose et saccharose}

Après comparaison avec une méthode enzymatique nous avons utilisé la méthode phénol-acide sulfurique mieux adaptée aux analyses en série $[19,20,21,22,23]$. 


\section{Matières azotées}

Les matières azotées totales sont dosées par micro-Kjeldahl.

\section{Cendres - Matières sèches}

Les cendres sont déterminées par calcination à $550^{\circ} \mathrm{C}$ et les teneurs en matière sèche par dessiccation à l'étuve à $100 \pm 2^{\circ} \mathrm{C}$, de $5 \mathrm{ml}$ de lait.

\section{Calcium}

Le calcium a été dosé par absorption atomique avec un appareil Varian Tectron AA6 suivant le protocole de Brulé, Maubois et Fauquant [24].

\section{Phosphore}

L'analyse du phosphore a été faite par la méthode colorimétrique F.I.L. $\mathrm{n}^{\circ} 42$ [25].

\section{Viscosité}

La mesure de la viscosité a été réalisée avec un viscosimètre à cylincires co-axiaux Haake RV 11.

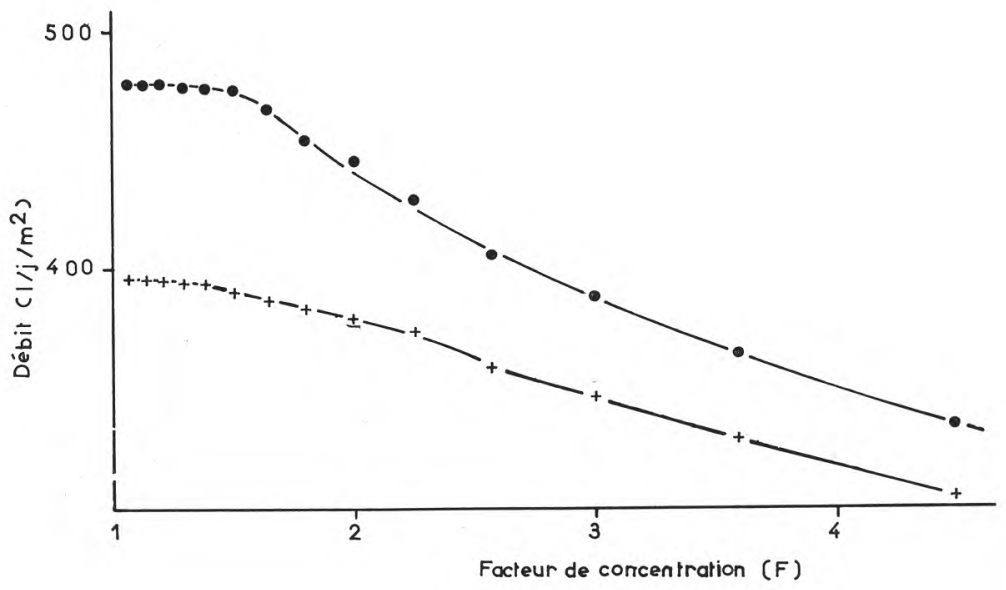

fig. 1

Débit d'ultrafiltration en fonction du facteur de concentration $\left(-\bullet-50^{\circ} \mathrm{C} ;-+-60^{\circ} \mathrm{C}\right)$ 


\section{RESULTATS}

\section{Choix de la température d'ultrafiltration}

Le tableau 1 rassemble les résultats obtenus à $50^{\circ} \mathrm{C}$. La quantité d'ultrafiltrat recueillie par jour et par $\mathrm{m}^{2}$ de membrane reste constante lorsque le facteur de concentration ne dépasse pas 1,2; elle diminue ensuite régulièrement.

La teneur en matières azotées totales (M.A.T.) de l'ultrafiltrat diminue légèrement à mesure que l'ultrafiltration progresse. Ces substances ne sont pas précipitables dans l'acide trichloracétique (T.C.A.) à 12 p. 100 de concentration finale [26].

$\mathrm{Au}$ contraire les teneurs en lactose et en calcium restent cons tantes et le $\mathrm{pH}$ ne varie pratiquement pas.

A $60^{\circ} \mathrm{C}$ le débit d'ultrafiltration est plus faible qu'à $50^{\circ} \mathrm{C}$ (fig. 1), et il décroit ensuite comme à $50^{\circ} \mathrm{C}$ à mesure que la concentration du rétentat augmente.

Les pourcentages d'élimination des différents composés sont à peu près identiques pour les deux températures. La seule différence importante porte sur le pourcentage de calcium éliminé qui est inférieur de 4 p. 100 à $60^{\circ} \mathrm{C}$.

\section{Choix du facteur de concentration}

L'analyse du tableau 2 montre que l'utilisation d'un facteur de concentration $\mathrm{F}$ égal à 5 donne un produit dont la teneur en lactose est environ le tiers de celle du lait du commerce et correspond à une concentration en lactose dans la phase aqueuse de 10,94 p. 100, assez faible pour qu'il n'y ait pas cristallisation de ce sucre, ce que confirment la dégustation et l'observation microscopique du produit après $45 \mathrm{j}$ de stockage à $9^{\circ} \mathrm{C}$.

Sa teneur en cendres est environ 2,5 fois plus faible que celle du lait commercialisé et sa viscosité est nettement inférieure (tab. 3). Cette teneur en cendres peu élevée est partiellement compensée par un meilleur équilibre entre le calcium et le phosphore puisque le rapport entre ces deux éléments est en effet de 1,4 plus élevé que celui d'un lait frais ou d'un lait écrémé sucré courant.

\section{Choix des barèmes de pasteurisation}

Dans le tableau 3 nous avons indiqué la viscosité et les pourcentages en calcium et lactose éliminés pour les laits écrémés concentrés sucrés préparés par ultrafiltration des laits écrémés et pasteurisés 30 secondes à 90,100 et $115^{\circ} \mathrm{C}$ et du lait cru. 
TABLEAU 1. - Résultats d'ultrafiltration à $50^{\circ} \mathrm{C}$

\begin{tabular}{|c|c|c|c|c|c|c|c|c|c|c|c|c|c|c|c|c|}
\hline & Lait & $\mathrm{U}_{1}$ & $\mathrm{U}_{2}$ & $\mathrm{U}_{3}$ & $\mathrm{U}_{4}$ & $\mathrm{U}_{5}$ & $\mathrm{U}_{6}$ & $\mathrm{U}_{7}$ & $\mathrm{U}_{8}$ & $\mathrm{U}_{9}$ & $\mathrm{U}_{10}$ & $\mathrm{U}_{11}$ & $\mathrm{U}_{12}$ & $\mathrm{U}_{13}$ & $\mathrm{U}_{14}$ & $\begin{array}{l}\text { Réten- } \\
\text { tat }\end{array}$ \\
\hline Volume (ml) & 180 & 10 & 10 & 10 & 10 & 10 & 10 & 10 & 10 & 10 & 10 & 10 & 10 & 10 & 10 & 40 \\
\hline Temps (mn, s) & & 10 & 20 & 30 & 40,05 & 50,12 & 60,2 & 71,56 & 84,03 & 96,4 & 111,05 & 129,15 & 147,30 & 170,15 & 199,45 & 199,45 \\
\hline Débit $\left(\mathrm{l} / \mathrm{j} / \mathrm{m}^{2}\right)$ & & 477 & 477 & 477 & 476,2 & 475,7 & 475,2 & 466,5 & 454 & 445,2 & 429,4 & 406,1 & 388,4 & 364,3 & 334,7 & \\
\hline $\mathrm{F}$ & & 1,06 & 1,13 & 1,2 & 1,29 & 1,38 & 1,5 & 1,64 & 1,8 & 2 & 2,25 & 2,57 & 3 & 3,6 & 4,5 & 4,5 \\
\hline $\mathrm{pH}$ & 6,72 & & & & & & & & & & & & & & & 6,7 \\
\hline M.A.T. (g/l) & 35,14 & 1,71 & 1,61 & 1,61 & 1,61 & 1,51 & 1,51 & 1,45 & 1,43 & 1,43 & 1,43 & 1,43 & 1,4 & 1,4 & 1,4 & 151,84 \\
\hline M.A.T. (p. 100) & 100 & 0,27 & 0,52 & 0,78 & 1,03 & 1,27 & 1,51 & 1,74 & 1,97 & 2,19 & 2,42 & 2,64 & 2,86 & 3,09 & 3,31 & 95,89 \\
\hline Lactose $(\mathrm{g} / \mathrm{l})$ & 46,04 & 45,38 & 46,26 & 45,71 & 46,04 & 45,71 & 45,38 & 46,59 & 46,04 & 45,38 & 45,71 & 45,38 & 45,38 & 46,26 & 45,71 & 45,38 \\
\hline Lactose (p. 100) & 100 & 5,47 & 11,05 & 16,56 & 22,11 & 27,62 & 33,09 & 38,71 & 44,26 & 49,73 & 55,24 & 60,71 & 66,18 & 71,26 & 77,27 & 21,9 \\
\hline Calcium (g/l) & 1,33 & 0,45 & 0,43 & 0,42 & 0,41 & 0,41 & 0,42 & 0,41 & 0,42 & 0,42 & 0,41 & 0,42 & 0,43 & 0,42 & 0,42 & 4,5 \\
\hline Calcium (p. 100) & 100 & 1,88 & 3,67 & 5,42 & 7,13 & 8,83 & 10,58 & 12,29 & 14,04 & 15,79 & 17,5 & 19,25 & 21,04 & 22,79 & 24,54 & 75,19 \\
\hline
\end{tabular}




\section{TABLEAU 2}

Composition des laits écrémés concentrés sucrés (L.E.C.S.) préparés par ultrafiltration et du lait écrémé concentré sucré du commerce

\begin{tabular}{|c|c|c|c|c|c|c|c|}
\hline Produits $\mathrm{F}$ & $\begin{array}{l}\text { L.E.C.S. } \\
\text { commerce }\end{array}$ & $\begin{array}{l}\text { L.E.C.S. } \\
2,57\end{array}$ & $\begin{array}{l}\text { L.E.C.S. } \\
3,00\end{array}$ & $\begin{array}{l}\text { L.E.C.S. } \\
3,60\end{array}$ & $\begin{array}{l}\text { L.E.C.S. } \\
4,50\end{array}$ & $\begin{array}{l}\text { L.E.C.S. } \\
5,00\end{array}$ & $\begin{array}{l}\text { Lait de } \\
\text { départ }\end{array}$ \\
\hline E.S.T. $(\mathrm{g} / \mathrm{kg})$ & 728,64 & 411,36 & 451,53 & 533,51 & 572,93 & 614,80 & 88,00 \\
\hline Saccharose $(\mathrm{g} / \mathrm{kg})$ & 423,26 & 296,31 & 329,41 & 370,46 & 423,82 & 449,74 & 0,00 \\
\hline Sacc./Sacc. $+\mathrm{H}_{2} \mathrm{O}(\times 100)$ & 60,93 & 33,48 & 37,52 & 44,26 & 49,81 & 53,86 & 0,00 \\
\hline M.A.T. (g/kg) & 108,05 & 59,56 & 61,71 & 81,14 & 84,95 & 91,24 & 33,53 \\
\hline Lactose $(\mathrm{g} / \mathrm{kg})$ & 128,26 & 33,17 & 35,52 & 36,79 & 39,04 & 42,13 & 44,18 \\
\hline Lactose $\left(\mathrm{g} / 100 \mathrm{~g} \mathrm{H}_{2} \mathrm{O}\right)$ & 47,27 & 5,64 & 6,48 & 7,89 & 9,14 & 10,94 & 4,84 \\
\hline Cendres $(\mathrm{g} / \mathrm{kg})$ & 21,97 & 5,11 & 6,69 & 6,98 & 7,50 & 9,52 & 7,88 \\
\hline $\mathrm{pH}$ & 6,50 & 6,72 & 6,72 & 6,72 & 6,72 & 6,72 & 6,75 \\
\hline Couleur & brun & blanc & blanc & blanc & blanc & blanc & blanc \\
\hline
\end{tabular}




\section{TABLEAU 3}

Caractéristiques des différents laits concentrés

\begin{tabular}{l|c|c|c}
\hline \multicolumn{1}{c|}{ Produit } & $\begin{array}{c}\text { Viscosité } \\
\mathrm{cp}\end{array}$ & $\begin{array}{c}\text { Calcium } \\
\text { éliminé } \\
\text { (p. 100) }\end{array}$ & $\begin{array}{c}\text { Lactose } \\
\text { éliminé } \\
\text { (p. 100) }\end{array}$ \\
\hline Lait écrémé concentré sucré du commerce & $6900^{*}$ & & \\
Lait concentré issu de lait cru & 13,11 & 24,22 & 78,31 \\
Lait concentré issu de lait pasteurisé à $90^{\circ} \mathrm{C}$ & 24,44 & 20,74 & 67,19 \\
Lait concentré issu de lait pasteurisé à $100^{\circ} \mathrm{C}$ & 37,15 & 20,0 & 63,47 \\
Lait concentré issu de lait pasteurisé à $115^{\circ} \mathrm{C}$ & 56,95 & 18,77 & 62,5 \\
\end{tabular}

* D'après Webb B. H. [2].

Les différents produits apparaissent comme des fluides non newtoniens. L'augmentation de viscosité est directement liée à la température de pasteurisation alors que les pourcentages de lactose et de calcium éliminés diminuent à mesure que le traitement thermique devient plus important. Cette diminution peut s'expliquer respectivement par l'augmentation de la viscosité et une insolubilisation plus importante du calcium. Malgré cet accroissement de la viscosité les produits obtenus restent encore très fluides par rapport au lait écrémé concentré sucré du commerce.

\section{DISCUSSION}

Les résultats indiquent que l'on peut fabriquer du lait écrémé concentré sucré suivant le schéma suivant :

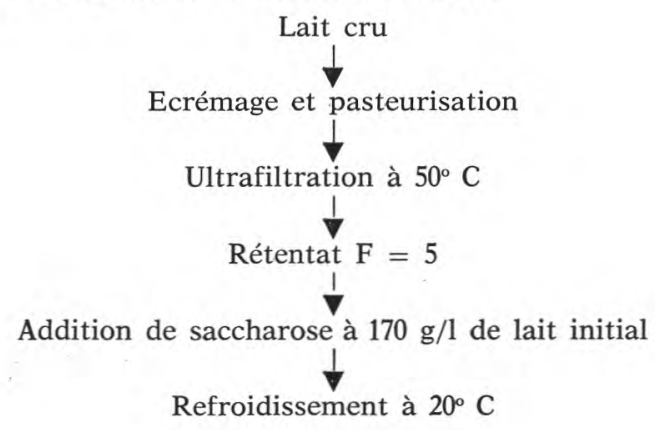


La température de $50^{\circ} \mathrm{C}$ convient parfaitement car elle donne un débit relativement important et permet de travailler pendant un temps limité qui réduit les risques bactériologiques et minimise les problèmes économiques [27]. Nos résultats concordent avec ceux de Pompei et al. [28] qui concluent que la meilleure température de concentration est $50^{\circ} \mathrm{C}$. Pour des températures inférieures en effet, les temps d'ultrafiltration sont assez longs pour favoriser le développement bactérien et, pour des températures supérieures, proches de $60^{\circ} \mathrm{C}$, il existe un risque important de dénaturer les protéines. Les auteurs précisent toutefois que le temps de séjour à $50^{\circ} \mathrm{C}$ doit être inférieur à 2 h., ce qui correspond à la phase de latence des germes thermophiles.

Le produit préparé avec un facteur de concentration égal à 5 est assez voisin du lait écrémé sucré concentré commercial en ce qui concerne le pourcentage en protéines et la teneur en saccharose. Il en diffère par son humidité, sa viscosité très faible et son rapport saccharose $\times 100$

saccharose + eau

qui est inférieur à la limite souhaitable de 60 p. 100 ,

seuil d'inhibition du développement bactérien. Il présente aussi un faible taux de lactose $(11$ p. 100$)$, un rapport $\mathrm{Ca} / \mathrm{P}$ relativement élevé $[1,4]$, et se caractérise par l'absence de brunissement enzymatique.

La faible teneur en lactose se traduit par une absence de cristallisation quelle que soit l'allure de la réfrigération du concentré et contribue à éviter la dénaturation des protéines du lait responsable du « thickening » pendant la conservation à basse température, phénomène étroitement lié à cette cristallisation $[29,30,31$, $32,33]$.

La diminution de la quantité de calcium dans le concentré joue également dans ce sens. D'après Wildasin et Doan en effet [34], le calcium soluble est nécessaire à la floculation de la caséine lors du stockage. D'après ces auteurs, la quantité de calcium à éliminer pour retarder, voire inhiber les floculations, serait de 10 p. $100 \mathrm{du}$ calcium total, quantité nettement inférieure à celle qu'élimine le traitement d'ultrafiltration $(18,77$ p. 100).

L'augmentation de 1,3 à 1,4 du rapport $\mathrm{Ca} / \mathrm{P}$ dans le lait écrémé concentré sucré préparé par ultrafiltration peut entraîner une amélioration de la qualité nutritionnelle de ce dérivé laitier en favorisant l'assimilation du phosphore [35].

Enfin, l'absence de couleur brune du lait concentré par ultrafiltration, témoin d'une meilleure conservation de la lysine et d'une valeur nutritionnelle accrue, constitue un avantage commercial non négligeable [36]. 


\section{v. CONCLUSION}

Le lait concentré sucré fabriqué par ultrafiltration présente de nombreux avantages :

- une faible teneur en lactose qui permet d'éviter l'apparition d'une texture sableuse et qui, conjointement avec la diminution de la teneur en calcium, réduit ou supprime les risques de floculation des protéines lors du stockage;

- une viscosité très peu élevée qui peut faciliter sa mise en œuvre ultérieure;

- une augmentation du rapport $\mathrm{Ca} / \mathrm{P}$ et de la quantité de lysine restant disponible;

- une coloration blanche qui peut être un argument commercial.

Cependant, à côté de ces avantages le produit concentré présaccharose $\times 100$

sente l'inconvénient d'avoir un rapport — trop faible

saccharose + eau

qui, joint à un taux de cendres peu élevé et un $\mathrm{pH}$ voisin de 6,7, peut entraîner une mauvaise conservation. Il est vraisemblable qu'une augmentation de la pression osmotique serait nécessaire pour pallier ce défaut, par addition de fructose ou de saccharose partiellement hydrolysé par exemple.

\section{R é s u m é}

L'utilisation de l'ultrafiltration pour la fabrication du lait concentré sucré permet d'éviter la texture sableuse dans le produit.

Les conditions de température et de concentration du procédé ont été précisées ainsi que ses inconvénients : faible viscosité et pression osmotique insuffisante, et ses avantages : risques d'épaississement réduits, absence de texture sableuse, rapport $\mathrm{Ca} / \mathrm{P}$ plus favorable, absence de coloration.

\section{S u m m a r y}

The presence of sandiness in swetened condensed milk can be avoided by the use of ultrafiltration during fabrication.

Details of temperature and concentration conditions of the products during processing are given. The disadvantages of the product are : low viscosity from the commercial point of view, insufficient osmotic pressure, and its advantages : less risk of thickening, absence of sandiness, more favorable $\mathrm{Ca} / \mathrm{P}$ relationship and absence of browning. 


\section{Bibliographie}

[1] Pien (J.) (1975). - Physico-chimie des laits concentrés. Troisième partie. La Technique Laitière, 30, 19-23.

[2] WebB (B. H.) (1970). - In: By products from milk. The Avi Publishing Company, 83-123.

[3] HaAse (G.), Nickerson (T. A.) (1966). - Kinetics reactions of $\alpha$ and $\beta$ lactose, I. mutarotation. J. Dairy Sci., 49, 127-132.

[4] Nickerson (T. A.), MOORE (E. V.) (1972). - Solubility interrelations of lactose and sucrose. J. Food Sci., 37, 60-61.

[5] Patel (K. N.), Nickerson (T. A.) (1970). - Influence of sucrose on the mutarotation velocity of lactose. J. Dairy Sci., 53, 1654-1658.

[6] Nickerson (T. A.), MOORE (E. V.) (1974). - Factors influencing lactose crystallization. J. Food Sci., 57, 1315-1318.

[7] TWIEG (W. C.), Nickerson (T. A.) (1968). - Kinetics of lactose crystallization. J. Dairy Sci., 51, 1720-1724.

[8] Nickerson (T. A.), Patel (K. N.) (1972), - Crystallization in solutions supersatured with sucrose and lactose. J. Food Sci., 37, 693-697.

[9] Nickerson (T. A.), Haase (G.) (1966). - Kinetics reactions of $\alpha$ and $\beta$ lactose. A. crystallization. J. Dairy Sci., 49, 757-760.

[10] Nickerson (T. A.), Lim (S. G.) (1974). - Effect of various alcohols on lactose. J. Food Sci., 57, 1320-1323,

[11] Nickerson (T. A.) (1977). - Alterations in the ratio of $\alpha$ to $\beta$ lactose co-crystallized from organic solvants. J. Food Sci., 42, 1066-1068.

[12] Jelen (P.), Coulter (S. T.) (1973). - Effects of supersaturation and temperature on the growth of lactose crystals. J. Food Sci., 38, 1182-1185.

[13] Jelen (P.), Coulter (S. T.) (1973). - Effects of certain salts and other whey substances on the growth of lactose crystals. J. Food Sci., 38, 1186-1189.

[14] Thurbly (J. A.), SitnaI (O.) (1976), - Lactose crystallization: investigation of some process alternatives. J. Food. Sci., 41, 43-47.

[15] Nickerson (T. A.) (1965). - In: Fundamentals of dairy chemistry. The Avi Publishing Company, 224-257.

[16] KosIKowsKi (F. V.), WIERZBICKI (L. E.) (1973). - Lactose hydrolysis in pasteurized milk by saccharomyces lactis lactase. J. Food Sci., 56, 146-148.

[17] Kisza (J.), Switka (J.), Krum (A.), Surazynski (A.) (1973). - Essai d'utilisation de la $\beta$-galactosidase pour la fabrication du lait condensé sucré. Le Lait, 53, 430-439.

L18] Roger (L.), Thapon (J.L.), Maubois (J. L.) (1976). - Hydrolyse du lactose contenu dans l'ultrafiltrat de lait ou de lactosérum en réacteur enzymatitique à membrane. Le Lait, 56, 56-75.

[19] Dubois (M.), Gilles (K. A.), Hamilton (J. K.), Rebers (P. A.) Smith (F.) (1956). - Colorimetric method for determination of sugars and related substances. Analy. Chem., 28, 350-356.

[20] Lawrence (A. J.) (1968). - The determination of lactose in milk products. The Aust. J. Dairy Tech., 23, 103.

[21] Birch (G. G.), Mwanglewa (O. M.) (1974). - Colorimetric determination of sugars in sweetened condensed milk products. J. Sci. Food Agric., 25, 1355-1362. 
[22] Tshibangu (M.) (1977). - Les caractéristiques organoleptiques et aromatiques et le brunissement enzymatique du jus de banane. Université des Sciences et Techniques du Languedoc. D.E.S. de Sciences Alimentaires, Montpellier.

[23] Laboratoires Mannheim-Boehringer. - Dosage du lactose et du galactose dans les milieux alimentaires. Fiche analytique, réf. 176303.

[24] Brulé (G.), Maubois (J. L.), Fauquant (J.) (1974). - Etude de la teneur en éléments minéraux des produits obtenus lors de l'ultrafiltration du lait sur membrane. Le Lait, 54, 600-611.

[25] PIEn (J.) (1969). - Dosage du phosphore dans le lait. Le Lait, 49, 175-188.

[26] Fenton-May (R. J.), Hill Jr (C. G.), Amundson (C. H.), Lopez (M. H.), Auclair (P. D.) (1972). - Concentration and fractionation of skim-milk by reverse osmosis and ultrafiltration. J. Dairy Sci., 55, 1561-1566.

[27] Peri (C.) (1975). - Maîtrise du développement microbien dans les systèmes à membranes. Séparations par membranes, échanges d'ions et cryoconcentration dans l'industrie alimentaire. Apria, Paris.

[28] Pompei (C.), Resmini (P.), Peri (C.) (1973). - Skim-milk protein recovery and purification by ultrafiltration. Influence of temperature on permeation rate and retention. J. Food. Sci., 38, 867-870.

[29] Tumerman (L.), Fram (H.), Cornely (K. W.) (1954). - The effect of lactose crystallization on protein stability in frozen concentrated milk. J. Dairy Sci., 37, 830-839.

[30] Rose (D.), Tessier (H.) (1956). - Effect of heat and of prefreezing storage on the stability of frozen milk. Can. J. Technol., 34, 139-144.

[31] Desai (J. D.), Nickerson (T. A.), Jennings (W. G.) (1961). - Stability of frozen milk. J. Dairy Sci., 44, 215-221.

[32] Wells (P. R.), Leeder (J. G.) (1963). - Changes related to casein precipitation in frozen concentrated milk. J. Dairy Sci., 46, 789-798.

[33] Nickerson (T. A.) (1964). - Changes in concentrated milk during frozen storage. J. Food Sci., 29, 443-447.

[34] Wildasin (H. L.), Doan (F. J.) (1951). - Some additional influences affecting the stability of concentrated milk in frozen storage. J. Dairy Sci., 34, 438-444.

[35] Gueguen (L.) (1971). - La composition minérale du lait et son adaptation aux besoins minéraux du jeune. Ann. Nut. Ali:, 25, A 335-A 381.

[36] de Vuyst (A.), Vervack (W.), Charlier (H.), Jadin (V.) (1972). - La teneur en lysine totale et disponible du lait, des fromages et de produits de boulangerie ayant subi un traitement thermique. Le Lait, 52, 444-453. 